\title{
On Evidence Supporting a Deterministic Process of Bacterial Growth
}

\author{
By A. L. KOCH \\ Departments of Biochemistry and Microbiology, College of Medicine, \\ University of Florida, Gainesville, Florida, U.S.A.
}

(Received 20 April 1965)

\begin{abstract}
SUMMARY
Recent evidence supporting a proposed model (Koch \& Schaechter, 1962) for the control of bacterial cell division is reviewed. Calculations from the published work of others are presented which show that the standard deviation of length of time between a cell division and $N$ th cell division does not increase, at least up to $N=9$. This finding implies that each cell has an excellent clock, which is handed to the daughters without significant error, and that the observed fluctuations in age of cells at division are largely due to an additional fluctuation associated with cell division, but not timing it. In terms of our model, it is strong support for the deterministic growth of cell constituents and the equipartition of cell constituents at division. An expansion of the original model is considered which accounts for the difference between the original model and the finding regarding the correlation coefficients of the ages of mothers and daughters and that between sisters, and the skewed nature of the agedistribution curve. In the new version of the model, the assumption that there are random fluctuations in the critical size at division is replaced by the assumption that the fluctuations are not completely random but that there is a moderately positive correlation between the sizes of divisions of successive generations.
\end{abstract}

Several years ago we proposed (Koch \& Schaechter, 1962) a model for bacterial cell division which took into account a number of the essential features of the statistical aspects of cell division as well as some of the known facts in the biochemistry of growth. To bridge the gap between the stochastical nature of cell division and the deterministic processes of protein synthesis, etc., the model had four basic postulates. First, that increase of bacterial constituents was substantially deterministic even at the single cell level. Explicitly, it was assumed that growth was exponential. Second, that there was a critical size which the cell must attain in order that division might ensue. Third, that there are random fluctuations, either about this critical time or a variable lag in the length of time when cell division would be apparent to the observer. Fourth, that cell division partitions the maternal cell, yielding daughters of identically the same size under certain conditions and nearly of the same size under other conditions. This model then could account for a number of the properties of growing populations observed by others, as well as experimental results from our own laboratory (Schaechter, Williamson, Hood \& Koch, 1962).

Recently, mathematical consequences of this model have been greatly augmented

Vol. 42, No. 3 was issued 6 May 1966 
by the work of Powell (1964). He has extended calculations based on this model to larger values of fluctuation and to fluctuation obeying more arbitrary distributions than were considered in our original publication or than seem to apply to the enteric bacteria.

However, of more vital import to this model would be further experimental justification of the four postulates on which it is based. Since the model was presented, two papers have appeared which contain data which we believe strongly support the concept that a highly deterministic process underlies the growth of micro-organisms and, in addition, that under certain conditions cell division leads to a precise equipartition of the cell contents.

A critical test for the simultaneous validity of both these assumptions, in that it would obviate the almost unavoidable experimental errors, would be the finding that standard deviation (s.D.) of the total time elapsed between one cell division and the cell division of all the $N$ th generation descendants would not increase with increasing $N$. Thus, independent of the details controlling the act of cell division, if the growth rate constant for protoplasm were constant at any time for all the cells, and if cell division divides the cell precisely in half, the variance for the interval between successive divisions should be the same, depending only on the chance fluctuations that determine the initial size of the first cell and the fluctuations that terminate the last division.

In our experimental work we did not make experiments over more than four generations because we felt that many cell divisions on a small region of a block of agar would lead to crowding which would interfere with the growth process and vitiate the conclusions. However, Kubitschek (1962) and Hoffman \& Frank (1965) successfully made such experiments; in the former case to the fifth generation and in the latter case to the fourteenth generation. Data were presented in both their papers which permit computation of the desired quantities. These are shown in Table 1. It can be seen that the s.D. of total elapsed time from the division of the maternal cell on to the fifth generation in the case of Kubitschek's experiment and on to the ninth generation in the case of the Hoffman \& Frank experiments proceeds without significant increase. This is true even though the microphotographs show that the organisms were extremely crowded by the end of the ninth generation. In the latter set of experiments, the overall mean interdivision time is not precisely given but from other data must be of the order of 21 min., so that with a coefficient of variation of $20 \%$, the s.D. for a single interdivision time is about $4 \mathrm{~min}$., which is not much less than the 5.69 min. fluctuation for 9 generations. This means that fluctuations in generation rate, together with the imperfections in equipartition at cell division, are much less than the coefficient of variation of the interdivision time divided by $\sqrt{ } N$, and thus are less than $20 \% / \sqrt{ } 9$, or $6 \%$ in a single generation.

This lack of increase in the s.D. with $N$ is a strong support for the idea that the fluctuation is entirely due to a random chance at first division initiating the parental cell and to the random fluctuation terminating the last division, no matter how many intervening divisions with their concomitant fluctuations are involved. Thus, it follows that the growth must be highly deterministic and that, in addition, cell division under these experimental conditions is a very precise process, dividing the cell into very nearly identical daughters. The relationship for the increase of cellular constituents under usual laboratory conditions at the single cell level must 


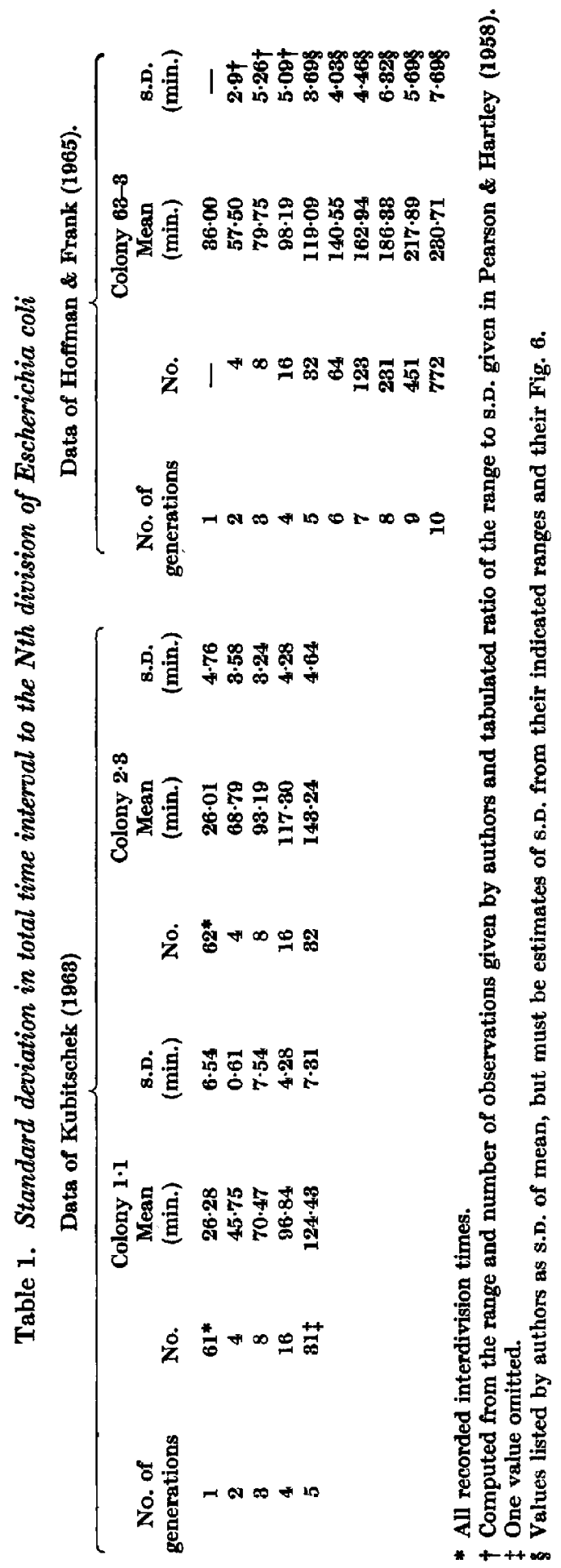


be very nearly a pure exponential. This is stressed by the recent findings that extend the concept that DNA synthesis is essentially continuous at the single cell level (Pachler, Schaechter \& Koch, 1965) and that there is exponential increase in DNA and other cell constituents within the cell cycle, as shown by the refined experiments of Cummings (1965) with synchronized organisms.

On the other hand, some of the predictions of the model are simply not generally obeyed. It is often found that the mother-daughter correlation coefficient is almost never as negative as $\mathbf{- 0 \cdot 5}$, that the sister-sister correlation is generally larger than +0.5 , and that the generation time distribution is positively skewed, in significant disagreement with the original model for precise equipartition at cell division. In fact, these were the findings in the experiments of Kubitschek quoted above. If it is assumed that these differences are not due to experimental artifacts, the original model must be refined. To do so, we must reject the concept of random fluctuation in the size of cells at division and replace it by a fluctuation in the critical size which is retained for a period extending over two or three generations. This assumption implies a moderate positive correlation in the sizes of cells at division of mother and daughter cells, as is the case (Errington, Powell \& Thompson, 1965; Koch, calculations on unpublished data of Kubitschek). Thus, a deviation in generation time from the mean in one generation may not be made up on the average in the next generation, but is made up in the next two or three generations. This is a contribution equivalent to short-term memory that would tend to make sister-sister correlation somewhat higher and the mother-daughter correlation somewhat lower (more positive) than predicted by the original explicit model and which could account for the skewed generation time distribution as well.

To see this qualitatively, imagine that the critical size for all the cells in the culture is not random at all but varies periodically and systematically. For this limiting case, the sister-sister correlation coefficient would be +1 , and the motherdaughter coefficient anywhere between +1 and -1 , depending on the relationship between the doubling time and the period of the fluctuation. If the variation in the critical size is small compared to its mean, as we have shown, the generation time distribution will be normal. If a small amount of such a systematic fluctuation is 'mixed in' with a random fluctuation determining the critical size, then it is evident that the sister-sister correlation coefficient will tend to be greater than +0.5 and the mother-daughter correlation coefficient will (in general) tend to be greater than $-0 \cdot 5$. The generation time distribution would still be normal.

The generation time distribution will be positively skewed if the persistent fluctuations are of a particular type. For example, if random fluctuations occur which quickly lower the critical size and are then followed by a slow return to normal, then the age distribution will be positively skewed. That the critical size is under physiological control is evident from the variation in cell size under different growth conditions. While such a model is required by the present data, further elaboration must await measurement of the distribution of the amounts of cell constituents amongst cells at the instant of cell division. A possible mechanism for the control of cell division which has these properties will be presented (Koch, in preparation). 
Work in the author's laboratory is supported by the National Cancer Institute CA-07404-02, National Institutes of Health, USPHS. The author wishes to thank Dr' H. E. Kubitschek for hospitality and extensive criticism, and Dr H. Hoffman for his confirmation that the author had correctly interpreted the published data of Hoffman \& Frank.

\section{REFERENCES}

Cummings, D. J. (1965). Macromolecular synthesis during synchronous growth of Escherichia coli в/r. Biochim. biophys. Acta, 95, 341.

Errington, F. P., Powele, E. O. \& Thompson, N. (1965). Growth characteristics of some Gram-negative bacteria. J. gen. Microbiol. 39, 109.

Hoffman, H. \& Frank, M. E. (1965). Synchrony of division in clonal microcolonies of Escherichia coli. J. Bact. 89, 513.

Koch, A. L. \& Schaechter, M. (1962). A model for statistics of the cell division process. J. gen. Microbiol. 29, 435.

KUBitscheK, H. E. (1962). Normal distribution of cell generation rate. Exp. Cell Res. 26, 439.

Pachler, P. R., Schaechter, M. \& Koch, A. L. (1965). Continuity of DNA synthesis in Escherichia coli. J. molec. Biol. $11,650$.

Pearson, E. S. \& Hartuey, H. O. (1958). Biometrika Tables for Statisticians, vol. I, p. 164. Table 20, Moment constants of the mean deviation and of the range. Cambridge University Press.

Powell, E. O. (1964). A note on Koch and Schaechter's hypothesis about growth and fission of bacteria. J. gen. Microbiol. 37, 231.

Schaechter, M., Williamson, J. P., Hood, J. R. Jun., \& Koch, A. L. (1962). Growth, cell and nuclear divisions in some bacteria. $J$. gen. Microbiol. $29,421$. 